\title{
The organisation and structure of inflammatory bowel disease services for children and young people in the UK in 2010: significant progress but still room for improvement
}

\author{
Michael P Fitzgerald, ${ }^{1}$ Sally G Mitton, ${ }^{2}$ Aimee Protheroe, ${ }^{3}$ \\ Michael Roughton, ${ }^{3}$ Richard Driscoll, ${ }^{4}$ Ian D R Arnott, ${ }^{5}$ Richard K Russell ${ }^{1}$
}

\begin{abstract}
'Department of Paediatric Gastroenterology, Yorkhill Hospital, Glasgow, UK ${ }^{2}$ Department of Paediatric Gastroenterology, St Georges Hospital, London, UK

${ }^{3}$ Clinical Effectiveness and Evaluation unit (CEEu), Royal College of Physicians of London, London, UK

${ }^{4}$ Crohn's and Colitis, St Albans, UK

${ }^{5}$ Gastrointestinal Unit, Institute of Genetics and Molecular Medicine, University of Edinburgh, Western General Hospital, Edinburgh, UK
\end{abstract}

\section{Correspondence to}

Dr Richard K Russell, Department of Paediatric Gastroenterology, Yorkhil Hospital, Dalnair Street, Glasgow G3 8SJ, UK; richardrussell@nhs.net

Received 28 June 2012 Revised 16 July 2012 Accepted 16 July 2012
To cite: Fitzgerald MP, Mitton SG, Protheroe A, et al. Frontline Gastroenterology 2013, 4, 25-31.

\begin{abstract}
Background Inflammatory bowel disease (IBD) is becoming more common in children. While treatment options remain limited the appropriate organisation and delivery of services are an integral part of good care.

Methods All eligible UK paediatric sites were invited to submit data for organisation of paediatric IBD services as of 1 September 2010. Comparison, when relevant, was made with the previous paediatric audit (2008) and the concurrently running adult audit.
\end{abstract}

Results 24/25 (96\%) of sites submitted data. The median number of patients managed and the median number of new IBD (ulcerative colitis and Crohn's disease only) cases per annum was 178 (IQR 136-281) and 32 (IQR 23-50), respectively. There was an increase in the IBD workforce including whole-time equivalent (WTE) IBD nurses (1.0 vs 1.5 WTE nurses, $\mathrm{p}=0.02$ ). 1023 patients 16 years and younger were looked after in the 202 adult sites who submitted data; only 78/202 sites indicated they cared for 16-year-old and younger children; approximately half of these 78 sites had ageappropriate support facilities. Most paediatric sites have access to urgent endoscopy (83\%)، telephone advice (100\%) and urgent clinic appointments (91\%). Most sites did not have: shared care pathways with primary care $(74 \%)$, annual reviews $(71 \%)$, real time patient management systems (83\%) and research network trial participation (78\%)

Conclusions Many aspects of paediatric IBD care in the UK are good and have shown significant improvement over recent years. There are areas in need of further change and specific regional and national action plans should address identified deficiencies before any future audit of paediatric and adult IBD services.

\section{INTRODUCTION}

Inflammatory bowel disease (IBD) is an umbrella term encompassing a variety of gastrointestinal inflammatory disorders, most notably Crohn's disease (CD) and ulcerative colitis (UC). Both are chronic, debilitating diseases with poorly understood aetiologies and no known cure. ${ }^{12}$ Although they have specific differences $\mathrm{UC}$ and $\mathrm{CD}$ are categorised together as they share many common features. Both diseases follow unpredictable relapsing and remitting courses, with significant variation in the pattern and complexity of symptoms between individuals. Half of all IBD patients will require gastrointestinal surgery during their lifetime, and extensive disease may engender life-threatening complications. $^{3} 4$ Conservative estimates for caring for the estimated $240000 \mathrm{IBD}$ patients in the UK cost the NHS in excess of $£ 1$ billion per annum, with these calculations made largely in a pre-biological era and when incidence and prevalence rates were lower. ${ }^{5} 6$

IBD is a significant paediatric disease, with approximately $25 \%$ of cases presenting in childhood. ${ }^{7}$ There is considerable geographical variation in the incidence of paediatric IBD, with rates ranging from 11.4/100 000/year in Canada to 0.25/ 100 000/year in Spain. ${ }^{8-10}$ The most up-to-date UK data come from a recent 
Scottish study that reported rates of 7.82/100 000/ year in children under 16 years of age. ${ }^{11}$ The same study showed an overall $76 \%$ increase in paediatric IBD cases between the periods $1990-5$ and 2003-8. ${ }^{11}$

IBD affects growing children very differently to mature adults, and consequently paediatric IBD requires a different approach in terms of treatment and management. ${ }^{12}$ Paediatric IBD patients are at particular risk of complications arising from malnutrition. ${ }^{12}$ Malnutrition may lead to growth failure and pubertal delay, ${ }^{13}$ which may result in diminished final adult height, ${ }^{14}$ reduced self-esteem ${ }^{15}$ and osteoporosis. ${ }^{16}$ Repeated corticosteroid therapy may also negatively impact growth potential and bone density. ${ }^{16}$ Anxiety and depression are also features of childhood disease and affected children will require age-specific treatment and therapy. IBD can impact greatly on the psychosocial development of adolescents, who may experience family conflict, trouble socialising with peers, medical adherence problems and absences from school. ${ }^{15} 1718$

As we currently have neither treatments to cure, nor interventions to prevent IBD, the goal for clinicians must be to optimise the care of these patients, in doing so clinical remission may be promoted and maintained and as a consequence quality of life is maximised. There is good evidence that clinical audit, with appropriate intervention and reassessment, is an effective method of improving the quality of care provision in chronic diseases. ${ }^{19-21}$

The results of the second round of the UK paediatric IBD audit are discussed and compared allowing direct comparison between paediatric IBD services in 2010 against those in 2008 as well as against nationally recognised standards of care.

\section{METHODS}

In 2008 the first national UK paediatric IBD audit was commissioned by collaboration between the relevant professional groups, patients' organisations and fundholders. ${ }^{22}$ The specific aims of the UK IBD audit as set out at the inception of the first adult audit in 2006 are listed in table 1.

The initial paediatric audit carried out in 2008 measured both the organisation and process of paediatric IBD care in the UK, against presumptive standards agreed by the UK IBD audit steering group based around the previously published British society of Gastroenterology guidelines. ${ }^{23}$ In 2009, the IBD standards were published following on from the IBD audit (box 1). The paediatric IBD audit was repeated in 2010, with this time the initial phase focusing solely on the organisation of paediatric IBD services (a separate audit on the provision of care to IBD patients in the UK is completed, the results of which were made available to participating sites in early 2012). The IBD audit steering group developed a dataset to measure paediatric IBD services at eligible
Table 1 The aims of the IBD audit at its inception

Aim 1 Assess current structure and organisation of care for IBD

Aim 2 Assess processes and outcomes of care delivery (inpatient and outpatient) in IBD

Aim 3 Enable trusts to compare their performance against national standards

Aim 4 Identify resource and organisational factors that may account for observed variations in care

Aim 5 Facilitate, develop and institute an intervention strategy to improve quality of care

Aim 6 Repeat the audit to prove that change has occurred

Aim 7 Establish measures for healthcare services to use to compare quality of IBD services

Aim 8 Develop a sustainability programme to maintain quality of care

$\mathrm{IBD}$, inflammatory bowel disease.

sites, against the IBD standards retaining some but not all of the original data structure from the first organisational audit. Data were entered via a secure online web tool, with each site having a unique site code.

\section{Box 1 The IBD standards}

\section{Standard A: High quality of clinical care}

- High quality, safe and integrated clinical care for IBD patients based on multidisciplinary team working and effective collaboration across NHS organisational structures and boundaries

\section{Standard B: Local delivery of care}

- Care for IBD patients that is delivered as locally as possible, but with rapid access to more specialised services when needed

Standard C: Maintaining a patient-centred service

- Care for IBD patients that is patient centred, responsive to individual needs and offers choice of clinical care and management when possible and appropriate

\section{Standard D: Patient education and support}

- Care for IBD patients that assists patients and their families in understanding IBD and how it is managed, and that supports them in shared decisionmaking and achieving the best quality of life possible within the constraints of the illness

\section{Standard E: information technology and audit}

- An IBD service that uses information technology effectively to support patient care and so to optimise clinical management through data collection and audit

\section{Standard F: Evidence-based practice and research}

- A service that is knowledge based and actively supports service improvement and research 
The data presented in this report are from the 2010 audit 'Report of the results for the national organisational audit of paediatric IBD services in the UK'. ${ }^{24}$ Comparative data were also analysed against the concurrent adult IBD organisational audit as well as the previous paediatric audit carried out in 2008 .

An eligible site was defined as a hospital or trust/ health board with a specialist paediatric gastroenterology unit within it. ${ }^{24}$ All eligible UK sites were then invited to participate and submit data pertaining to their paediatric IBD services as of $1^{\text {st }}$ September 2010. Some organisational questions related to admissions and operations for IBD that took place during the 12-month period from September 2009 to 31 August 2010. The figures for IBD patients included those with CD and UC only, with patients who had a diagnosis of IBD-unclassified (IBDU) ${ }^{25}$ excluded from the audit.

Descriptive data are presented with medians and IQR. Comparison between medians was performed using the Mann-Whitney test and with categorical variables using the $\chi^{2}$ test or Fisher's exact test as appropriate.

\section{RESULTS}

Twenty-five sites in the UK were deemed eligible for inclusion in the 2010 UK paediatric IBD audit, and 24 (96\%) of these sites submitted data (see appendix 1 for list of participating sites). Of the 24 sites, one site submitted a partly completed dataset. The full list of data items collected can be found at http://www.rcplondon. ac.uk/resources/inflammatory-bowel-disease-audit.

\section{Patient numbers and registers}

In the 2010 audit the median number of patients managed by each site was 178 (IQR 136-281). The median number of new IBD patients per annum was 32 (IQR 23-50). The number of new patients compared to the total number of patients was $18 \%$ of the total. Adult sites managed a median of 788 (500-1497) patients and saw a median of 60 (34-115) new patients per annum, with new patients comprising $8 \%$ of the total. The proportion of new patients seen in paediatric services was significantly higher $(\mathrm{p}<0.001)$. No directly comparative data were available from the previous audit to assess whether overall patient numbers were changing.

In generating or estimating these numbers a register of IBD patients was maintained in 78\% (18/23) of sites; however, $50 \%(12 / 24)$ of sites had to estimate the number of IBD patients managed by their service, suggesting some registers were not up to date, or were used for other purposes such as monitoring of immunosuppressive drug use. Using local registers $57 \%(13 / 23)$ of sites captured clinical data about their patients and $17 \%(4 / 23)$ used a real-time system to support management of their IBD patients.
The median number of UC inpatients admitted per site over a year was 10 (IQR 4-17). For CD this was 19 (IQR 7-32). Of these a median number of two UC (IQR 0-3) and four CD (IQR 1-6) patients underwent surgery during their admission.

\section{Workforce and site configuration}

Most $(83 \%, 20 / 23)$ clinical teams had an IBD lead who was usually a consultant paediatric gastroenterologist (17/20). The median number of whole-time equivalent (WTE) paediatric consultant gastroenterologists per site increased from 2 in 2008 to 2.2 (IQR 1.4-3.6) and the median number of WTE paediatric surgeons increased from 4.5 to 5.5 (IQR 4.2-7) ( $p>0.05$ for both).

There was a statistically significant increase in the median number of WTE paediatric gastroenterology/ IBD nurses per site increasing from one in 2008 to $1.5(0.9-2)$ in $2010(\mathrm{p}=0.02)$; the number of sites with a nurse increased from $61 \%$ previously $(14 / 23)$ to $83 \%(19 / 23)(\mathrm{p}=0.10)$. In 2010 a total of $92 \%$ $(22 / 24)$ of paediatric IBD sites had access to a nutritional support team, 67\% (16/24) had access to a psychologist and $83 \%(20 / 24)$ of sites had linked with a designated colleague in adult gastroenterology who had a recognised interest in adolescent IBD.

The number of dedicated paediatric IBD wards in the UK increased from six in 2008 to eight in 2010. The ratio of easily accessible toilets to beds on these designated wards fell from a median of 4 in 2008 to 3.3 in 2010 . The majority of these toilets are mixed $\operatorname{sex}(88 \%)$.

\section{'Paediatric' care in adult sites}

The audit of adult IBD services revealed that 39\% (78/202) of adult sites care for IBD patients aged 16 years and under. Across all 202 adult sites 1023 patients (680 CD and $343 \mathrm{UC}$ ) aged 16 years and under were looked after in adult IBD services in the 1-year period between September 2009 and 2010, including $97 \mathrm{CD}$ patients and 38 UC patients who underwent surgery. Of the 78 sites who said they cared for paediatric patients $46 \%(36 / 78)$ had a specific paediatric to adult transition policy, 47\% (37/78) had a surgeon with suitable paediatric experience, $53 \%(41 / 78)$ had an endoscopy area with ageappropriate facilities, $56 \%(44 / 78)$ had a trained/ experienced paediatric endoscopist and 68\% (53/78) had an anaesthetist with paediatric training.

\section{Organisation of IBD services}

Amongst sites that took part in both rounds of the audit, the number of sites that have local guidelines for the management of acute severe colitis has increased from 10 in 2008 to 14 in $2010(\mathrm{p}=0.24)$. Eighty-three per cent (20/24) of sites have regular timetabled meetings to discuss IBD patients, and these take place weekly in 65\% (13) of these 20 sites. The 
median waiting time for an urgent clinic appointment for suspected IBD patients is 7 days (5-11). In $83 \%$ $(20 / 24)$ of sites relapsed patients have access to endoscopy within $72 \mathrm{~h}$ of admission, and urgent colonic biopsy results are available within $48 \mathrm{~h}$. Seventy-one per cent (17/24) of sites, however, do not have formal arrangements for annual outpatient review. When performed the annual review most commonly consisted of bloods tests (examining inflammatory and nutritional indices) together with measures of growth and pubertal development.

Twenty-six per cent $(6 / 23)$ of sites reported having shared care protocols for paediatric IBD patients with general practitioners (GPs), but all sites were found to share results of patients' disease activity and treatment changes with GPs.

\section{Patient services}

Eighty-seven per cent of sites (20/23) provide a pathway for the patient to discuss their treatment with the IBD team, with $91 \%$ (21/23) providing written information to the patient about who to contact in the event of a relapse. All sites have arrangements to expedite specialist review of relapsed patients. Specialist review of relapsed patients occurs within 7 days at $91 \%(21 / 23)$ of sites and within 5 days at $65 \%(15 / 23)$ of sites. All sites provided telephone access to an IBD specialist, with $96 \%$ (22/23) responding within $48 \mathrm{~h} ; 26 \%(6 / 23)$ of sites offered patients a choice about different ways for follow-up beyond the traditional method of an outpatient review.

Ninety-six per cent $(22 / 23)$ of sites provided specific information to patients with newly diagnosed IBD, with 43\% (10/23) providing patients with a written care plan. Sixty-five per cent (15/23) of sites provide educational opportunities for patients and their carers. Translation services were available in $96 \%$ $(22 / 23)$, with $39 \%(9 / 23)$ providing written information on IBD in different languages. Nine out of 23 sites have open forum meetings for patients.

\section{Surgery}

The majority of sites $(75 \%, 18 / 24)$ performed ileo-anal pouch operations, performing a median of one (IQR 0-3) operation per year.

\section{Education and research}

During the specified 12-month period of the audit, paediatric gastroenterology/IBD nurse specialists received a median of 2 days $(0-5)$ IBD-specific training. Twenty-two per cent (5/23) of sites entered patients into Medicines for Children Research Network (MRCN) supported trials, with the same number of centres holding annual review days of service.

\section{DISCUSSION}

Paediatric IBD services in the UK are sufficiently organised for the vast majority of centres to provide meaningful data and so participate in the national audit. This audit provides evidence of service improvement in many areas since the initial benchmarking audit carried out 2 years previously.

All 25 UK sites eligible for inclusion in the 2010 UK paediatric IBD audit registered to participate, and ultimately 24 sites $(96 \%)$ submitted data. The high participation rate emphasises the good organisational practice and dedication of the local teams involved in the audit and also confers a great degree of confidence on the quality of data generated.

The report highlights that in 2010 paediatric IBD services in the UK continue to be consultant led. Between 2008 and 2010 there was an encouraging rise in the number of specialist medical and nursing staff available to treat paediatric IBD patients. In particular, there has been a considerable increase in the median number of WTE paediatric gastroenterology/ IBD nurse specialists at sites. Although they are a relatively new development, IBD nurses are proving a vital part of the healthcare team involved in the delivery of care to IBD patients. There is an increasing awareness of the important role they can play in the provision of a comprehensive multidisciplinary package of care. Published studies haves been suggested that in adult populations IBD nurse specialists improve access to and availability of IBD services as well as often being better equipped to deal with patient education and psychosocial issues. ${ }^{26}$ An uncontrolled study by Nightingale et $a l^{27}$ conducted on an adult IBD population suggested that the employment of IBD nurse specialists can result in a reduction in hospital visits and inpatient stay, along with an increase in remission and overall patient satisfaction. Supportive data have also been generated from the UK IBD audit to show the improvement in care associated with centres that have IBD nurses compared to those that do not. ${ }^{28}$

The adult UK IBD audit found that a substantial number of adult hospitals care for paediatric patients and that many of these sites have neither the appropriately trained staff nor the age-appropriate facilities to care for children. Paediatric sites had significantly better access to dietetic services and psychology. Standard A12 of the IBD standards stipulates that young people with IBD should be looked after in an age-appropriate setting with support from professionals with suitable paediatric experience. ${ }^{29}$ It is difficult to envisage paediatric IBD patients having their height, not to mention their pubertal status, monitored in an adult IBD clinic, although there is some evidence to the contrary it was published some time ago. ${ }^{30}$ Children, as with patients of all ages, deserve to be cared for in facilities suitable for their requirements and by appropriately trained healthcare 
professionals. Resolving this situation will require the engagement of adult and paediatric services at both a national and local level. ${ }^{31} 32$

Despite $78 \%$ of sites indicating that they maintain a register of IBD patients, $50 \%$ of sites could only give an estimate when asked how many IBD patients are managed by their service. Current and reliable demographic data, cataloguing the incidence, prevalence and location of paediatric IBD in the UK, are essential if we are to plan on a national level how best to provide services into the future for an ever growing IBD population. Proof of the increased number of paediatric IBD patients should translate to increased resource allocation and safeguard provision of services to our patients. The development of a UK-wide realtime IBD database would encourage and facilitate local participation.

Patents with diagnoses of IBDU were excluded from the audit. This group may represent a significant proportion of paediatric IBD patients. $^{33}$ By excluding these patients from the audit a valuable opportunity to understand in more detail the incidence, prevalence and service requirement of this poorly characterised patient population has been lost. Future audits should look to include this patient group.

The audit found that only $22 \%$ of sites reported entering children in to MCRN trials; this is particularly disappointing especially considering that the equivalent figure from the adult IBD audit was 35\%. There is a recognised deficit and urgent need for research into paediatric IBD as a result of the fact that the majority of existing scientific information regarding IBD pathophysiology and therapeutic approaches are findings from adult studies, animal models or cell culture studies. ${ }^{34}$ Children with IBD are an excellent population in which to study microbial and immune interactions relevant to disease pathophysiology as they potentially have fewer confounders such as longterm antibiotics, corticosteroids and smoking compared to adults with the disease. Given the lifelong nature of IBD our experience suggests that parents of patients are often eager to enter their children in trials in the hope of an eventual cure for the disease. It is difficult to envisage significant future breakthroughs in our understanding of the pathophysiology of IBD without a present upsurge in the number of studies involving paediatric patients.

In general, the standards of services in paediatric gastroenterology sites are in line with those set out in 'Service standards for the care of people who have IBD 2009'. When compared to the report from the 2008 IBD audit, there is a considerable improvement in the standard of care available to paediatric IBD patients, especially when one considers the relative short time period between the two reports. However, all children should have access to the full complement of multidisciplinary services, be treated in an age-appropriate facility by suitably trained staff plus a considerable improvement needed in the number of clinical trials involving paediatric patients.

\section{What is already known on this topic}

- The national paediatric IBD audit was first undertaken in 2008 and was repeated in 2010

- There has been a marked improvement in the provision of services to paediatric IBD patients, in particular in the number of specialist medical and nursing staff available to treat these patients.

- There is still room for improvement in:

Communication with GPs.

The number of children looked after in adult services and the provisions made for them, including patients with unclassified IBD.

All paediatric gastroenterology units should have up-to-date real-time patient databases.

Paediatric gastroenterology units' participation in MRCN supported trials.

- The challenge for paediatric gastroenterology units is to take on board the above recommendations, to optimise further the service they provide and to continue to participate in future national paediatric IBD audits.

\section{What this study adds}

The national paediatric IBD audit was first undertaken in 2008 and was repeated in 2010.

- There has been a marked improvement in the provision of services to paediatric IBD patients, in particular in the number of specialist medical and nursing staff available to treat these patients.

There is still room for improvement in: Communication with GPs.

The number of children looked after in adult services Including patients with IBDU

How might it impact on clinical practice in the foreseeable future'

Institution of real-time patient databases

Increased paediatric gastroenterology unitsâ participation in MCRN supported trials.

- Continue to participate in future national paediatric IBD audits

- Joint paediatric and adult initiatives to support in particular teenagers with IBD

Acknowledgements The UK IBD audit steering group would like to thank and acknowledge all who have participated in the piloting and development of the IBD audit.

Funding The UK IBD Audit (2010) was commissioned by the Healthcare Quality Improvement Partnership with additional financial support from NHS Quality Improvement Scotland. The 2008 audit was funded by the Health Foundation.

The IBD team at Yorkhill Hospital Glasgow are supported by the Catherine McEwan Foundation and Yorkhill IBD fund. RKR is supported by an NHS Research Scotland career fellowship award. 
Contributors RKR, SGM, IDRA, AP and RD designed and/or modified the organisational dataset for the audit; MR performed the statistical analysis; MPF and RKR wrote the manuscript and all authors critically reviewed and amended the manuscript and all agreed on the final version.

\section{Competing interests None.}

Provenance and peer review Commissioned; internally peer reviewed.

\section{REFERENCES}

1 Casati J, Toner BB, de Rooy EC, et al. Concerns of patients with inflammatory bowel disease: a review of emerging themes. Dig Dis Sci 2000;45:26-31.

2 Korzenik J. Past and current theories of etiology of IBD: toothpaste, worms, and refrigerators. J Clin Gastroenterol 2005;39(4 Suppl. 2):S59-65.

3 Langholz E, Munkholm P, Davidsen M, et al. Course of ulcerative colitis: analysis of changes in disease activity over years. Gastroenterology 1994;107:3-11.

4 Munkholm P, Langholz E, Davidsen M, et al. Intestinal cancer risk and mortality in patients with Crohn's disease. Gastroenterology 1993;105:1716-23.

5 Molodecky NA, Soon IS, Rabi DM, et al. Increasing incidence and prevalence of the inflammatory bowel diseases with time, based on systematic review. Gastroenterology 2012;142:46.

6 Luces C, Bodger K. Economic burden of inflammatory bowel disease: a UK perspective. Expert Rev Pharmacoecon Outcomes Res 2006;6:471-82.

7 Griffiths A. Specificities of inflammatory bowel disease in childhood. Best Pract Res Clin Gastroenterol 2004;18:509-23.

8 Benchimol EI, Fortinsky KJ, Gozdyra P, et al. Epidemiology of pediatric inflammatory bowel disease: a systematic review of international trends. Inflamm Bowel Dis 2011;17:423-39.

9 Benchimol EI, Guttmann A, Griffiths AM, et al. Increasing incidence of paediatric inflammatory bowel disease in Ontario, Canada: evidence from health administrative data. Gut 2009;58:1490-7.

10 Fernandez GN, Bousono GC, Ramos PE, et al. Inflammatory bowel disease in pediatric patients in Asturias, Spain, (19932000): epidemiology and clinical features [Spainish]. Acta Pediatr Esp 2004;62:466-72.

11 Henderson P, Hansen R, Cameron FL, et al. Rising incidence of pediatric inflammatory bowel disease in Scotland. Inflamm Bowel Dis 2012;18:999-1005.

12 Kelsen J, Baldassano RN. Inflammatory bowel disease: the difference between children and adults. Inflamm Bowel Dis 2008;14(S2):S9-11.

13 Ballinger A, Savage M, Sanderson I. Delayed puberty associated with inflammatory bowel disease. Pediatr Res 2003;53:205-10.

14 Markowitz J, Grancher K, Rosa J, et al. Growth failure in pediatric inflammatory bowel disease. J Pediatr Gastroenterol Nutr 1993;16:373-80.

15 Engstrom I. Inflammatory bowel disease in children and adolescents: mental health and family functioning. J Pediatr Gastroenterol Nutr 1999;28:S28-33.
16 Gokhale R, Favus MJ, Karrison T, et al. Bone mineral density assessment in children with inflammatory bowel disease. Gastroenterology 1998;114:902-11.

17 Griffiths AM, Nicholas D, Smith C, et al. Development of a quality-of-life index for pediatric inflammatory bowel disease: dealing with differences related to age and IBD type.J Pediatr Gastroenterol Nutr 1999;28:S46-52.

18 Ross SC, Strachan J, Russell RK, et al. Psychosocial functioning and health-related quality of life in paediatric inflammatory bowel disease. J Pediatr Gastroenterol Nutr 2011;53:480-8.

19 Jefferson IG, Swift PGF, Skinner TC, et al. Diabetes services in the UK: third national survey confirms continuing deficiencies. Arch Dis Child 2003;88:53-6.

20 Lelliott P, Wing J. A national audit of new long-stay psychiatric patients. II: Impact on services. Br J Psychiatry 1994;165:170-8.

21 Price LC, Lowe D, Hosker HSR, et al. UK National COPD Audit 2003: impact of hospital resources and organisation of care on patient outcome following admission for acute COPD exacerbation. Thorax 2006;61:837-42.

22 UK Inflammatory Bowel Disease Steering Group. UK IBD Audit 2008 (Executive Summary). London: Royal College of Physicians, 2009.

23 Carter MJ, Lobo AJ, Travis SP, et al. Guidelines for the management of inflammatory bowel disease in adults. Gut 2004;53(Suppl. 5):v1.

24 UK Inflammatory Bowel Disease Steering Group. Report of the results for the national organisational audit of paediatric inflammatory bowel disease services in the UK. London: Royal College of Physicians, 2011.

25 Silverberg M, Satsangi J, Ahmad T, et al. Toward an integrated clinical, molecular and serological classification of inflammatory bowel disease: Report of a Working Party of the 2005 Montreal World Congress of Gastroenterology. Can J Gastroenterol 2005;19(Suppl. A):5-36.

26 Younge L, Norton C. Contribution of specialist nurses in managing patients with IBD. Br J Nurs 2007;16:208-12.

27 Nightingale AJ, Middleton W, Middleton SJ, et al. Evaluation of the effectiveness of a specialist nurse in the management of inflammatory bowel disease (IBD). Eur J Gastroenterol Hepatol 2000;12:967-73.

28 Fernandez E, Kemp K. Impact of inflammatory bowel disease nurse specialist on quality of patient care and meeting strategic national aims. Gut 2012;61(Suppl. 2A):178.

29 http://www.ibdstandards.org.uk/ (accessed 14 Jun 2012).

30 Barton JR, Gillon S, Ferguson A. Incidence of inflammatory bowel disease in Scottish children between 1968 and 1983 marginal fall in ulcerative colitis, three-fold rise in Crohn's disease. Gut 1989;30:618-22.

31 The Scottish Government. Better Health, Better Care: National Delivery Plan for Children and Young People's Specialist Services in Scotland. Edinburgh: The Scottish Government, 2009.

32 Department of Health. Executive Summary, National Service Framework for Children, Young People and Maternity Services London: Department of Health, 2004.

33 Prenzel F, Uhlig H. Frequency of indeterminate colitis in children and adults with IBD-a metaanalysis. J Crohn's Colitis 2009;3:277-81.

34 Bousvaros A, Morley Fletcher A, Pensabene L, et al. Research and clinical challenges in paediatric inflammatory bowel disease. Dig Liver Dis 2008;40:32-8. 
APPENDIX 1.

PARTICIPATING SITES; NATIONAL

ORGANISATIONAL AUDIT OF PAEDIATRIC INFLAMMATORY BOWEL DISEASE SERVICES

IN THE UK

Addenbrooke's Hospital (Paediatric Gastro Unit)

Alder Hey Children's Hospital

Barts and the London Children's Hospital

Birmingham Children's Hospital

Bristol Royal Hospital for Sick Children

Children's Services, Chelsea and Westminster Hospital

Department of Child Health, University Hospital of

Wales

Great Ormond Street Hospital

Leeds General Infirmary (Paediatric Gastro Unit)

Leicester Royal Infirmary Children's Hospital

Morriston Hospital (Paediatric Gastroenterology)
North-East Scotland Paediatric Gastroenterology Network (Royal Aberdeen Children's Hospital, Ninewells Hospital and Raigmore Hospital combined) Oxford Children's Hospital

Royal Belfast Hospital for Sick Children

Royal Free Hospital (Paediatric Gastroenterology Unit)

Royal Hospital for Sick Children, Edinburgh

Royal Manchester Children's Hospital

Royal Victoria Infirmary Children's Services

Sheffield Children's Hospital

Southampton Children's Hospital

St George's Hospital (Paediatric Gastro Unit)

St Mark's Hospital (Paediatric Gastroenterology)

The Children's Hospital, Lewisham

Yorkhill Children's Hospital, Glasgow 